\title{
Superimposed Polymyositis in a Patient with Myeloperoxidase-Related Crescentic Glomerulonephritis
}

\author{
Shang-Feng Tsai ${ }^{1,2}$ and Jun-Li Tsai ${ }^{3^{*}}$ \\ ${ }^{1}$ Department of Medicine, Division of Nephrology, Taichung Veterans General Hospital \\ ${ }^{2}$ Department of Life Science, Tunghai University, Taichung, Taiwan \\ ${ }^{3}$ Cheng Ching General Hospital, Taichung, Taiwan
}

*Corresponding author: Jun-Li Tsai, MD, Cheng Ching General Hospital, Taichung, Taiwan, H-No. 118, Section 3, Chung-Kang Road, Taichung 407, Taiwan, Tel: 886-4-23592525, ext. 3034; Fax: 886-4-23594980; E-mail: chestnutlt@yahoo.com.tw

Rec date: Apr 28, 2014, Acc date: June 28, 2014, Pub date: July 5, 2014

Copyright: (c) 2014 Tsai SF, et al. This is an open-access article distributed under the terms of the Creative Commons Attribution License, which permits unrestricted use, distribution, and reproduction in any medium, provided the original author and source are credited.

\begin{abstract}
Renal involvement especially glomerulonephropathy in polymyositis or dermatomyositis is considered rare. Crescentic glomerulonephritis is even more so. Herein, we report a patient who was diagnosed with concurrent polymyositis and myeloperoxidase-related crescentic glomerulonephritis. We discuss the association between polymyositis or dermatomyositis and renal involvement, glomerulonephropathy, and crescentic glomerulonephritis. This is the first report of a case of polymyositis and myeloperoxidase-related crescentic glomerulonephritis with a clear temporal correlation.
\end{abstract}

Keywords: Myeloperoxidase-related crescentic glomerulonephritis; Polymyositis; Glomerulonephropathy

\section{Introduction}

The major presentation of polymyositis or dermatomyositis is the obvious muscle weakness and skin rash. The superimposed renal involvement had been previously considered as rare. However, the notion of its scarcity is incorrect after we performed this literature review focusing on renal involvement in polymyositis or dermatomyositis. The acute tubular injury is the most prevalent type of renal involvement followed by glomerular injury. Of all the glomerular injury, crescentic glomerulonephritis is the least common. This article will present the first case of pauci-immune related crescentic glomerulonephritis superimposed with polymyositis.

\section{Case Report}

A 72-year-old man was presented with kidney injury and gradual dysphagia for 2 months at the outpatient department. Uremia-related dysphagia was first excluded due to low blood urea nitrogen (BUN:59 $\mathrm{mg} / \mathrm{dL}$ ). Duodenoscopy revealed no mechanical obstruction. Patient later claimed about his muscle soreness. Biochemical data were as follows: creatinine kinase, $2472 \mathrm{U} / \mathrm{L}$; lactic dehydrogenase, $450 \mathrm{U} / \mathrm{L}$; aldolase, $21.7 \mathrm{U} / \mathrm{L}$; Jo-1, 139 units and anti-nuclear antibody, 1:160. Esophageal transit time was prolonged and electromyography also suggested myopathy. Myositis scan revealed diffuse myositis at four extremities. Polymyositis was highly suspected even without muscle biopsy. Malignancy surveys including chest X-ray (CXR), enteroscopy, and abdominal sonography were negative. We prescribed $100 \mathrm{mg}$ of hydrocortisone three times per day as his major treatment.

The patient's serum creatinine was increased from $0.8 \mathrm{mg} / \mathrm{dL}$ to 6.5 $\mathrm{mg} / \mathrm{dL}$ with hematuria and subnephrotic range of proteinuria. His immunological data disclosed negative double-strand DNA (dsDNA), normal proteinase 3, and high myeloperoxidase (MPO, 109.2 units) in the absence of anti-glomerular basement membrane (GBM) antibody. Furthermore, his renal biopsy showered cellular crescent (Figure 1) and mesangial proliferation. The immunofluorescence microscopy was negative for IgA staining and there were no full-house or doublecontour staining nor linear or granular staining over GBM, which suggest pauci-immune glomerulonephritis. Five days later, doublefiltration plasmapheresis (DFP) was administered 5 times per day. We did not administer methylprednisolone or cyclophosphamide pulse therapy to the patient owing to concern of sepsis. After five-time-DFP, we initiated hemodialysis (HD) due to high BUN $(107 \mathrm{mg} / \mathrm{dL})$, and metabolic acidosis $\left(13 \mathrm{meq} / \mathrm{L}\right.$ of $\left.\mathrm{HCO}_{3^{-}}\right)$. Soon after $\mathrm{HD}$, the patient went into shock and Klebsiella pneumoniae was found in blood and sputum with pneumonia over right lower lung. Two days later, the patient was expired due to refractory signs of shock regardless of piperacillin and tazobactam use.

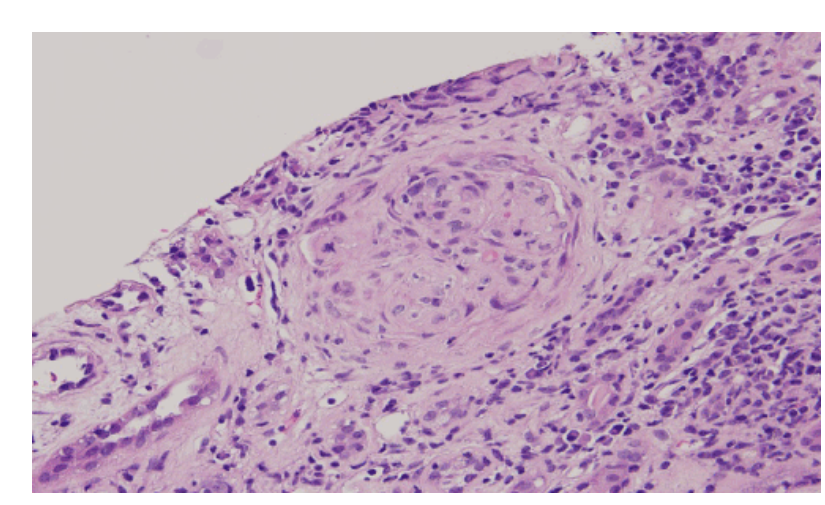

Figure 1: Renal biopsy in light microscopy staining for hematoxylin and eosin. The glomerulus shows necrotizing change with cellular crescent formation and ruptured Bowman's capsule. 


\section{Materials and Methods}

We performed an extensive literatures review from MEDLINE. Articles writing in all languages were included. There are only four cases with MPO-related crescentic glomerulonephritis in polymyositis/dermatomyositis. We had summarized the characteristics in Table 1.

\begin{tabular}{|l|l|l|l|l|l|}
\hline $\begin{array}{l}\text { Year } \\
\text { reported }\end{array}$ & gender & age & $\begin{array}{l}\text { Period (years) between } \\
\text { PM/DM and crescentic } \\
\text { glomerulonephritis }\end{array}$ & $\begin{array}{l}\text { Level of } \\
\text { MPO (EU) }\end{array}$ & outcome \\
\hline $1999[5]$ & female & 54 & 3 & 420 & uremia \\
\hline $2003[6]$ & female & 39 & 10 & 149 & uremia \\
\hline $2011[4]$ & male & 60 & 10 & 1280 & uremia \\
\hline $\begin{array}{l}2013 \\
\text { (present } \\
\text { study) }\end{array}$ & male & 72 & concurrent & 109.2 & uremia \\
\hline
\end{tabular}

Table 1: MPO-related crescentic glomerulonephritis in polymyositis/ dermatomyositis

\section{Discussion}

Renal involvement in polymyositis or dermatomyositis (PM/DM) had been considered as rare. However, according to a recent report of $14 \mathrm{PM} / \mathrm{DM}$ patients [1], its incidence rate is $25.1 \%$ (14 patients). These patients are categorized into myoglobulin-related acute tubular injury (ATI) and glomerulonephropathy. Here raises the questions of why renal involvement is seem as uncommon. In order to understand the reasons behind the issue, first we acknowledge that $\mathrm{PM} / \mathrm{DM}$ itself is a rare disease. Second, although ATI is common, it could easily be missed without meticulous checkup due to its self-limiting well prognosis. Third, most clinicians are reluctant to perform renal biopsy just to prove ATI. Therefore, if only we could precisely diagnose every ATI as renal involvement in $\mathrm{PM} / \mathrm{DM}$, the incidence rate will not be low. In the study by Yen et al. [1], 9 of the $14 \mathrm{PM} / \mathrm{DM}$ patients $(64.3 \%)$ with renal involvement had ATI but only 2 patients (14.3\%) had biopsy-proved glomerulonephropathy. The incident of glomerulonephropathy is still rare.

According to a literature [2], only 22 cases of PM/DM with glomerulonephropathy had been reported worldwide. Adding on our patient (23 patients), 12 patients had mesangial proliferative glomerulonephritis, 6 had membranous glomerulonephropathy, 4 had crescentic glomerulonephritis (CGN), and 1 patient had minimal change disease. We believe this scarcity is due to under diagnosis. Clinical manifestations of $\mathrm{PM} / \mathrm{DM}$ are more dominant than those with glomerulonephropathy and clinicians would usually first prioritize treating the more dominant symptoms of PM/DM. Patients' gomerulonephropathy would then be left partially treated and causeing the status of its under diagnosis. Our patient had mesangial proliferation, but IgA nephropathy or lupus nephritis was unlikely due to negative stain for IgA and lack of full-house staining pattern or dsDNA. CGN were also detected and it is even more uncommon currently.

There are only 4 reported cases of PM/DM with CGN [3]. To the best of our knowledge, this patient in our study is the $5^{\text {th }}$ case. They were all immune-complex related and what differs from our case to the others is that our patient's report shows pauci-immune, which has never been previously described in literatures. Realizing that complement deposits attack vascular endothelium in muscles, damage muscle capillaries, and attack glomerular membrane, we know they all act to compromise the protective mechanism of the capillaries. Therefore, the mechanism of the immune-complex-related CGN is reasonable and myoglobulin-anti-myoglobulin antibody-related IC has been reported. However, this case was diagnosed as MPO-related CGN. After extensive reviews, we found three more cases of MPOrelated CGN [4-6], but DM occurred at least 3 years before CGN. Correlation between MPO-related CGN and PM/DM is not yet clear. To the best of our knowledge, our case is the first attempt to describe a clear temporal association between MPO-related CGN and PM/DM (Table 1). Beforehand, concurrence was considered coincidental because there are at least 3 years elapsed between the onset of these two diseases. Henceforth, the likelihood of temporal correlation should be considered strong and clinicians should be aware of the signs of $\mathrm{PM} / \mathrm{DM}$ with renal function impairment. We highly suspect that the rarity of concurrence is mostly due to under-diagnosis, which may be caused by partial treatment of PM/DM with steroids.

\section{Conclusion}

Renal function involvement in $\mathrm{PM} / \mathrm{DM}$ is not as uncommon as most clinicians believe in the past. Most of the reported cases have involved ATI, and we should always keep in mind the possibility of concurrent MPO-related CGN and PM/DM.

\section{Learning Point for Clinicians}

Renal involvement in polymyositis/dermatomyositis is rare due to partial treatment and under diagnosis. Clinicians should take heed about crescentic glomerulonephritis and myeloperoxidase-related crescentic glomerulonephritis regarding its underestimated prevalence and treatment methods.

\section{References}

1. Yen TH, Lai PC, Chen CC, Hsueh S, Huang JY (2005) Renal involvement in patients with polymyositis and dermatomyositis. Int J Clin Pract 59: 188-193.

2. Takizawa Y, Kanda H, Sato K, Kawahata K, Yamaguchi A, et al. (2007) Polymyositis associated with focal mesangial proliferative glomerulonephritis with depositions of immune complexes. Clin Rheumatol 26: 792-796.

3. Tsunemi M, Ishimura E, Tsumura K, Shoji S, Sugimura T, et al. (1993) A case of crescentic glomerulonephritis associated with polymyositis. Nephron 64: 488-489.

4. Kawai H, Kitagawa W, Suzuki N, Maeda K, Suzuki K, et al. (2011) Myeloperoxidase-antineutrophil cytoplasmic antibody-related crescentic glomerulonephritis after treatment for clinically amyopathic dermatomyositis: a coincidental combination or not? Clin Exp Nephrol 15: 577-581.

5. Kushihata S, Hirabayashi A, Yorioka N (1999) ANCA-related glomerulonephritis during dermatomyositis. Jpn J Nephrol 41: 567 (Japanese abstract).

6. Iwabuchi M, Arakawa M, Hanakama M, Sasaki Y (2003) MPO-ANCA related glomerulonephritis at ten years after dermatomyositis. Jpn J Nephrol 45: 534 (Japanese abstract). 